CHAOS 15, 041101 (2005)

\title{
Introduction: Second Annual Gallery of Nonlinear Images (Los Angeles, California, 2005)
}

Bulbul Chakraborty

Department of Physics, Brandeis University, Waltham, Massachusetts 02454

Charles R. Doering

Department of Mathematics and Michigan Center for Theoretical Physics, University of Michigan, Ann Arbor, Michigan 48109-1109

Walter I. Goldburg

Department of Physics and Astronomy, University of Pittsburgh, Pittsburgh, Pennsylvania 15260

(Received 14 November 2005; published online 30 December 2005)

[DOI: $10.1063 / 1.2150155]$

In the following pages, the winning entries in the Gallery of Nonlinear Images are presented. The competition was held at the American Physical Society's March 2005 meeting in Los Angeles and was sponsored by the APS Topical Group on Statistical and Nonlinear Physics (GSNP). We thank the many members of the nonlinear and statistical community who participated in the event. We hope these images (and the videos that accompany some of them) will help to convey, in a striking and memorable way, the intricate beauty of nonlinear phenomena, and we encourage all our colleagues to submit their images to future Gallery com- petitions, which will be held at all subsequent APS March meetings.

As Guest Editors for this Gallery, we wish also to thank the American Institute of Physics and the Editors of Chaos for publishing the single-page versions of the entries in the printed and electronic versions of Chaos and for hosting the Gallery website at http://chaos.aip.org/gallery. We look forward to the third annual Gallery of Nonlinear Images competition, scheduled to be held at the 2006 APS March Meeting in Baltimore. 